between these two, but it can gradually be bridged, until speaking with ease and freedom becomes as spontaneous as was the original impulse to speech involving the stammer.

With regard to guidance of the speech itself, it may be of assistance to recall the analogy of a slow-motion film showing some perfect movement, such as the stroke of an expert tennis player, which elucidates normal rhythmic action. If our own speech be examined in an analogous way, the exact nature of the difference between normal and stammering speech qua speech is revealed. Having due regard to all that has been said on the matter of mental over-tension and emotional interference, a helpful mode of guidance may here be perceived.

It need hardly be said that the adequate treatment of this very difficult subject within the scope of an article of a few thousand words would be an utter impossibility. The most that I have been able to do is to give a few hints as to the nature of the disorder, and the more general principles by which one should be guided in taking up treatment.

Many years of special attention devoted to this most interesting of speech disorders, and the making and correcting (at-least in part) of, probably, most of the mistakes that can be made, during this apprenticeship, have convinced me that those best fitted to deal with it are those who have the widest perception as to possible lines of treatment, combined with the clearest perception of the principles involved; who are prepared to admit that, at least so far as we can now see, there can be no one panacea as regards technique in treatment for all stammerers; who are willing, wherever it seems advisable, to pass a patient on to another, and, perhaps even more important, are able to see when this is advisable; and who are glad to share with other workers along lines differing from their own, the pleasure, as well as the responsibility, of relieving the patient of his difficulty.

\title{
THE TREATMENT OF COMPOUND FRACTURES.
}

\author{
ALAN C. GAIRDNER, B.M., B.Ch.(Oxon.), F.R.C.S.(Eng.),
}

Medical Superintendent, St. George-in-the-East Hospital.

This article is a review of the various methods of treatment of compound fracture and a discussion of the problems which arise as the result of the opening of a fracture to the outside air.

A compound fracture is essentially one in which there is a solution of continuity of the skin over the fracture and this introduces two factors which are of the greatest importance in determining the final result. They are:-

(i) The introduction of infection around the fracture.

(ii) The escape of blood which in the closed fracture collects around the broken ends of the bone as a hæmatoma. Both these possibilities are of very great importance in modifying the processes of repair. 
The complications which may ensue on a compound fracture are bound up with these two factors: in some cases the first and in some the second is paramount. The complications may be:-

(i) Those of any severe lacerated wound, i.e. (a) shock, (b) severe infection of the soft tissues with abscess formation, gas gangrene, tetanus, etc.

(ii) Severe osteomyelitis with sequestration.

(iii) Delayed or non-union.

In all these complications, except, of course, in the case of shock, it has been the custom to regard infection as the dominating element and, indeed, in severe infections of the bone and soft parts this is so. But infection does not explain delayed or non-union in compound fractures where there is no demonstrable infection, where there is no sequestration and where the wound to the exterior heals by first intention. To explain this it is stated that infection is present but of so mild a degree as to produce no visible reaction and has merely interfered with the osteogenetic powers of the tissues. In such cases, however, it is probable that the major factor is the loss of the integrity of the hæmatoma surrounding the bone. It is the integrity of the hæmatoma, as recent researches have shown, which contributes very markedly to the bony union of the fracture for it is by deposition of calcium in its substance that fractures are united and consolidated.

From the above considerations it follows that in the treatment of a compound fracture, in addition to the adequate fixation of the fragments, two further principles must be adhered to:-

(i) The prevention of infection.

(ii) The preservation as far as possible of the hæmatoma surrounding the fracture.

Unfortunately, these two principles tend to be antagonistic as the best method of preventing infection is to remove all debris and blood clot, and to leave the wound as dry as possible in order to exclude any inanimate material in which bacteria may flourish and multiply. It is, however, possible to arrive at a compromise by studying each individual case as it is treated.

\section{Treatment (Orthodox).}

A compound fracture is of course a matter of emergency. Immediate treatment is always desirable, the emergency being only modified by the necessity for treating shock, but even then treatment should not be long delayed as the presence of an undressed wound and the pain of an unimmobilized fracture tend to perpetuate the shock. The patient must therefore be suitably anæsthetized and treatment proceeded with. This consists essentially in a wound toilet but the nature of this should vary with the type of fracture.

Compound fractures can be divided into two main classes. In the first there is a relatively large skin wound with considerable tissue laceration. In such cases the tissues and skin laceration are usually produced by the fracturing force such as the passage of a wheel over the limb in question. A thorough wound toilet should be performed. The skin edges should be excised and the dead and lacerated tissues removed. When such cleansing has been done a primary suture may be 
attempted or the wound may be packed. Antiseptics are innumerable, but probably the best for such purposes is flavine or some other of the analine compounds which are not toxic to the body tissues.

There is, however, a second class of compound fracture in which there is a puncture in the skin, which is usually quite small and produced by the sharp end of the bone perforating the skin from within. Often by the time these cases arrive for treatment this hole is closed with blood clot and is no longer bleeding. In these circumstances I consider that the best method of treatment is not the opening up of the wound with the performance of an extensive toilet but the immediate and firm suture of the skin in an attempt to maintain the integrity of the hæmatoma and reproduce as soon as possible the conditions of a closed fracture. It is my opinion that treatment of this kind does not increase the liability to infection and that it tends to render the occurrence of non-union less likely.

The toilet of the wound having been accomplished the next problem is the fixation of the fracture. The fracture must be completely reduced and if possible end to end apposition should be obtained. The methods of fixation are varied and may be classified as follows:-

(i) Wooden or metal splints fixed with bandages.

(ii) Extension apparatus.

(iii) Plaster of Paris with windows.

(iv) Completely closed Plaster of Paris.

There is no question that first-intention healing and minimal infection, as well as good final results, are best obtained by firm fixation. However, until recently it has been thought desirable to leave the site of the compound fracture exposed in order to allow of frequent dressing. Consequently, the first three methods have been generally adopted but from the point of view of firm fixation they all have great disadvantages. In the use of wooden splints firm fixation cannot be obtained and the removal of a part of the fixing bandages for dressing purposes produces frequent movement of the fracture and is uncomfortable for the patient. Extension apparatus is somewhat of an improvement on the first method but it is difficult to fit to a compound fracture, does not give firm fixation and always confines the patient to bed. Plaster of Paris with a window is more satisfactory as it gives adequate fixation with access for dressings. If, however, the wound suppurates, which is a relatively common occurrence, the discharge from the wound combined with the soaking by dressing lotions causes a disintegration of the plaster with loss of fixation. Moreover, rigid asepsis in dressing is rendered difficult, if not impossible, when operating through a small plaster window.

\section{Special Technique Recommended.}

In my opinion the method of choice is the closed complete plaster, for by this method adequate firm fixation is obtained and the reduction of the fracture is maintained. If first intention healing is attained, the further treatment does not differ from that of a closed (simple) fracture. If suppuration occurs, harm does not necessarily result. The work of Winnett and Orr has shown that no ill effects ensue from a wound being dressed with and bathed in its own discharges, and indeed wounds involving bone often appear to heal more readily if excluded from outside interference, although bathed in their own discharges. Moreover, by this technique there is no danger of the addition of a secondary infection which, even with the greatest care, is bound to occur when a wound is dressed daily for a prolonged period. 
The following method of fixation is therefore indicated. After the reduction and toilet of the fracture the wound is lightly dressed with gauze impregnated with vaseline which permits the free exit of any discharges. A close-fitting unpadded plaster is then applied direct to the skin. No window is cut. The limb is elevated on return to the ward in order to limit any swelling which may occur under the plaster. The plaster is left intact for six weeks. Should suppuration occur the discharge soaks into the plaster or escapes at either end. The odour may become strong but this usually causes no discomfort to the patient.

As a rule the patient has no pain in the limb after the application of the plaster, or at any rate only for a day or two, and he may be allowed to get about on crutches within a week even if the fracture is in the lower limb. After six weeks the plaster is removed, the limb cleansed and any stitches removed. If union has occurred the limb is protected with a light plaster splint for a further period varying with the site of the fracture.

If, however, union has not occurred a closed plaster is applied for another period of six weeks, at the end of which time the case is once more reviewed and any further treatment will depend on the condition of the lesion. Satisfactory bony union of course may have resulted, but on the other hand, the union may be fibrous or completely lacking, and the wound may be closed or open. Again, sequestration of part of the bone may have occurred.

Under no circumstances can any operative treatment such as bone grafting be considered at this early stage, for even if the wound is soundly healed operative interference is likely to cause a recrudescence of any residual infection.

Should examination by the X-rays reveal any sequestra these must be removed in order to bring about the closure of any sinuses.

If there is absolute non-union, a very rare occurrence, the limb should be placed in a complete plaster to avoid the occurrence of a deformity and later, when the wound has been satisfactorily healed for six months, an attempt may be made to obtain union by an autogenous bone graft.

If, however, fibrous union has occurred, or at any rate is of such a nature as to prevent deformity from gravity, massage and movements of the adjacent joints may be started while the fracture is protected by a light removable plaster. If the fracture is in the lower extremity the weight of the body must be kept off the limb, but the patient may be allowed to get about with a walking calliper-splint.

At this stage some movement and strain of slight degree around the fracture, as is given by massage and movement of the joints, may determine the formation of a bony union. This may occur quite quickly, firm union resulting within a month, and it is possible that this union is due to the formation of a hæmatoma around the fracture in which callus formation may take place. If, however, union does not occur within a year, an attempt must be made to obtain bony union by bone grafting, provided the wound has healed.

\section{Prognosis.}

The prognosis in compound fracture is always more serious than in simple fracture. In the lower extremity, even in the most favourable cases, a period of disability of 9 months may be expected while in more serious cases 18 months' disability may be anticipated, and in some cases persistent non-union may necessitate amputation of the limb. 